\title{
Establishment and validation of a guinea pig model for human congenital toxoplasmosis
}

\author{
Thomas Grochow ${ }^{1,2}$, Britta Beck ${ }^{1,2}$, Zaida Rentería-Solís², Gereon Schares ${ }^{3}$, Pavlo Maksimov³ ${ }^{3}$ Christina Strube ${ }^{4}$, \\ Johannes Seeger ${ }^{1}$, Lisa Raqué ${ }^{5}$, Reiner Ulrich ${ }^{6}$, Arwid Daugschies ${ }^{2}$ and Simone A. Fietz ${ }^{1 *}$
}

\begin{abstract}
Background: Toxoplasma gondii is an obligate intracellular parasite with a worldwide distribution. Congenital infection in humans and animals may lead to severe symptoms in the offspring, especially in the brain. A suitable animal model for human congenital toxoplasmosis is currently lacking. The aim of this study is to establish and validate the guinea pig as a model for human congenital toxoplasmosis by investigating the impact of the T. gondii infection dose, the duration of infection and the gestational stage at infection on the seroconversion, survival rate of dams, fate of the offspring, T. gondii DNA loads in various offspring tissues and organs and the integrity of the offspring brain.

Methods: Pregnant guinea pigs were infected with three different doses (10, 100, 500 oocysts) of T. gondii strain ME49 at three different time points during gestation (15, 30, 48 days post-conception). Serum of dams was tested for the presence of T. gondii antibodies using immunoblotting. T. gondii DNA levels in the dam and offspring were determined by qPCR. Offspring brains were examined histologically.

Results: We found the survival rate of dams and fate of the offspring to be highly dependent on the T. gondii infection dose with an inoculation of 500 oocysts ending lethally for all respective offspring. Moreover, both parameters differ depending on the gestational stage at infection with infection in the first and third trimester of gestation resulting in a high offspring mortality rate. The duration of infection was found to substantially impact the seroconversion rate of dams with the probability of seroconversion exceeding $50 \%$ after day 20 post-infection. Furthermore, the infection duration of dams influenced the T. gondii DNA loads in the offspring and the integrity of offspring brain. Highest DNA levels were found in the offspring brain of dams infected for $\geq 34$ days.

Conclusion: This study contributes to establishing the guinea pig as a suitable model for human congenital toxoplasmosis and thus lays the foundation for using the guinea pig as a suitable animal model to study scientific questions of high topicality and clinical significance, which address the pathogenesis, diagnosis, therapy and prognosis of congenital toxoplasmosis.
\end{abstract}

Keywords: Congenital toxoplasmosis, Toxoplasma gondii, Guinea pig, Animal model, Oocysts infection, Stage of gestation, Infection dose, Predilection site, Brain lesion

*Correspondence: simone.fietz@vetmed.uni-leipzig.de

${ }^{1}$ Institute of Veterinary Anatomy, Histology and Embryology, Faculty of Veterinary Medicine, University of Leipzig, Leipzig, Germany

Full list of author information is available at the end of the article

\begin{abstract}
Background
Toxoplasmosis is a zoonosis caused by Toxoplasma gondii, an obligate intracellular parasite with a worldwide distribution [1-3]. The global seroprevalence for $T$. gondii in adults is approximately $33 \%[4,5]$. The global incidence of $T$. gondii infection during pregnancy is $1.5 \%$ for women of childbearing age [5]. Congenital T. gondii infections are of clinical and economic importance in
\end{abstract}

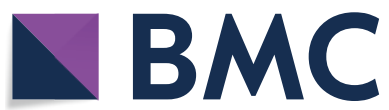

(c) The Author(s) 2021. Open Access This article is licensed under a Creative Commons Attribution 4.0 International License, which permits use, sharing, adaptation, distribution and reproduction in any medium or format, as long as you give appropriate credit to the original author(s) and the source, provide a link to the Creative Commons licence, and indicate if changes were made. The images or other third party material in this article are included in the article's Creative Commons licence, unless indicated otherwise in a credit line to the material. If material is not included in the article's Creative Commons licence and your intended use is not permitted by statutory regulation or exceeds the permitted use, you will need to obtain permission directly from the copyright holder. To view a copy of this licence, visit http://creativecommons.org/licenses/by/4.0/. The Creative Commons Public Domain Dedication waiver (http://creativeco mmons.org/publicdomain/zero/1.0/) applies to the data made available in this article, unless otherwise stated in a credit line to the data. 
pets and livestock, especially in small ruminants [6-8]. The definitive hosts of $T$. gondii are cats and other Felidae; all warm-blooded animals are intermediate hosts. T. gondii passes through several developmental stages in its life cycle $[9,10]$. Sexual reproduction of the parasite occurs in the intestinal epithelium of the definitive host. The resulting oocysts are shed in the feces [11]. Oral infection of an intermediate host results in rapid asexual multiplication of the acute (tachyzoite) stage [10, 12]. Tachyzoites can reach and infect all tissues of the host via the bloodstream, lymphatic system and peritoneal fluid. Due to the reaction of the immune system, the parasite is pushed back into the chronic stage, and intracellular tissue cysts containing bradyzoites are formed about 10-14 days after infection. Bradyzoites have a reduced metabolism, and cysts may persist for life in the organism, especially in the brain, retina, and skeletal and cardiac muscles [10,13-16]. Main routes of infection of the intermediate host are ingestion of food or water contaminated with sporulated oocysts and raw or insufficiently treated meat containing cysts. Given that the parasite crosses the placental barrier, T. gondii may be transmitted to the embryo/fetus during maternal parasitemia after a primary infection of the mother [1, 3, 17]. In adult healthy and immunocompetent humans, initial infection is usually asymptomatic. However, congenital infection may lead to severe symptoms in the offspring. The pathological changes occur particularly in the developing central nervous system $[9,18]$. In this regard, the clinical picture in humans depends on the time of infection during pregnancy, i.e. the earlier the time of infection, the more severe the symptoms caused by T. gondii. Infection in the first trimester may cause abortion. Microcephaly, retinochorioiditis and mental retardation are classic signs of a relatively severe course and may indicate infection in the second trimester. When infection occurs in the third trimester of pregnancy, cerebral calcifications and hepatosplenomegaly predominate $[19,20]$.

A suitable animal model for human congenital toxoplasmosis is currently not available. Mouse and rat appear to be less suitable because of their relatively short gestation period of approximately 20-22 days [21-23]. Moreover, both species are-in contrast to humans-primary altricial mammals and are therefore characterized by a low degree of maturation at the time of birth and the development of a hemotrichorial placenta, in which three layers of chorionic epithelium separate the maternal blood from the fetal capillaries [24]. Large domestic mammals such as ruminants and pigs are precocial mammals. However, as ruminants and pigs develop an epitheliochorial placenta, in which a single-layer chorionic epithelium borders the intact wall of the endometrium and underlying maternal capillaries, they appear to be less suitable as animal models for studying congenital infections [24]. The guinea pig is a precocial mammal, whose brain, similar to that of humans, is characterized by a relatively high degree of maturity at birth [25]. In analogy to the human cortex, neurogenesis in the guinea pig neocortex mainly occurs in the second trimester of gestation [26]. Moreover, it shows great similarities with humans regarding the placentation. Similar to humans, the guinea pig exhibits a haemomonochorial placenta, in which a single-layer, syncytial chorionic epithelium is in direct contact with the maternal blood. Furthermore, in both species, progesterone is produced by the placenta during pregnancy and the sexual cycle is characterized by a cyclically recurring estrus [27-29]. In addition, the guinea pig has a relatively long gestation period of approximately 66 days. Due to these characteristics, the guinea pig has been considered a suitable model animal for the study of human congenital toxoplasmosis [21, 22, 30]. Depending on the Toxoplasma strain, dose and mode of infection, toxoplasmosis in adult guinea pigs can be asymptomatic, but may also lead to myocarditis, myositis, encephalitis, pneumonia and hepatitis [21, 31, 32].

The aim of this study is to establish and validate the guinea pig as a model animal for human congenital toxoplasmosis by investigating the impact of the $T$. gondii infection dose, the duration of infection and the gestational stage at infection on the seroconversion rate, the survival rate of the dams, the fate of the offspring, the $T$. gondii DNA loads in various offspring tissues and organs and the integrity of the offspring brain. This study provides the basis for the proposed use of the guinea pig as an animal model for the investigation of scientific aspects of high medical importance, particularly the pathogenesis of congenital toxoplasmosis in humans.

\section{Methods \\ Animals}

Female Dunkin Hartley guinea pigs $(n=30)$ were obtained from Charles River Laboratories (Ecully, France) and housed in the animal-care facility of the Institute of Parasitology, Faculty of Veterinary Medicine, University of Leipzig, Leipzig, Germany. Animals were kept in groups of two in $50 \times 70 \times 50 \mathrm{~cm}$ wire mesh cages. Water supplemented with $400 \mathrm{mg} / \mathrm{l}$ ascorbic acid, standard diet pellets (Altromin Spezialfutter, Lage, Germany) and fresh vegetables were provided ad libitum. Room temperature was kept at $19-25^{\circ} \mathrm{C}$, and relative humidity averaged $55 \%$ $( \pm 10 \%)$. Light/dark cycle was set to 12:12.

\section{Mating and pregnancy examination}

Females $(n=30)$ were mated at the Institute of Parasitology, Faculty of Veterinary Medicine, University of 
Leipzig. Prior to breeding, their sexual cycle was synchronized as described previously [33]. Briefly, females were orally administered Altrenogest (Regumate ${ }^{\circledR}$ Equine $2.2 \mathrm{mg} / \mathrm{ml}$, MSD Tiergesundheit, Unterschleißheim, Germany) at $0.22 \mathrm{mg} / \mathrm{kg}$ body weight once a day for 15 days. Two days after the end of treatment, four females were housed together with one breeding ram for a total of 4 days to initiate natural mating. Successful pregnancy was determined by ultrasound using GE Logiq $400 \mathrm{CL}$ (pet mode, 7-10 MHz sample, GE Healthcare, Solingen, Germany). As a result, the day of successful mating is considered as day $0 \pm 2$ of pregnancy. During ultrasonographic examination, the reproductive system including ovary and uterus wall of all dams was examined. In addition, the vagina of all dams was examined by inspection.

\section{Serological investigation}

Blood samples of dams were collected from lateral saphenous vein immediately before infection and by intracardiac puncture at the respective end of trial. Samples were centrifuged $\left(2500 \times g, 10 \mathrm{~min}, 4{ }^{\circ} \mathrm{C}\right)$; serum was collected and analyzed using immunoblotting against $T$. gondii surface antigen p30 (SAG1) as described previously [34] with the modification that, instead of the peroxidase conjugated anti-mouse IgG, a peroxidase conjugated anti-guinea pig IgG $(\mathrm{H}+\mathrm{L})$ (Jackson Immunoresearch Laboratories, West Grove, USA) was used.

\section{Infection}

Toxoplasma gondii oocysts (Strain ME49) were obtained from the Institute of Parasitology, University of Veterinary Medicine Hannover, Germany. Seronegative cats were fed with meat supplemented with brain, muscle, liver and spleen of guinea pigs chronically infected with $T$. gondii. The presence of the parasite in the brain was confirmed by squash histology. Cat feces were purified as described previously [35], and isolated oocysts were stored in $2 \%$ sulfuric acid at $4{ }^{\circ} \mathrm{C}$ for $1.5-4$ months. Immediately before infection, the oocyst suspension was neutralized with $1 \mathrm{M}$ sodium hydroxide solution, and sporulated oocysts were quantified using a NeubauerImproved haemocytometer (Paul Marienfeld, LaudaKönigshofen, Germany). Oocysts were suspended in $500 \mu \mathrm{l}$ phosphate-buffered saline solution, and the suspension was applied orally using a $16 \mathrm{G}$ buttoned cannula (Henry Schein Dental, Langen, Germany).

Guinea pigs were randomly divided into a control group $(n=3)$ and three different infection groups: low$(n=9)$, medium- $(n=9)$ and high-dose group $(n=9)$. Dams of the low-dose group were administered 10 oocysts, dams of the medium-dose group were given 100 oocysts, and dams of the high-dose group were administered 500 oocysts on gestation day $15(n=3), 30(n=3)$ or $48(n=3)$, respectively. Animals of the control group were given $500 \mu \mathrm{l}$ phosphate-buffered saline solution only. All T. gondii infections were carried out in parallel.

\section{Euthanasia and dissection}

Dams and their corresponding litter were killed by intraperitoneal injection of $500 \mathrm{mg} / \mathrm{kg}$ pentobarbital sodium immediately after birth, abortion, resorption or upon the appearance of severe neurological or other clinical symptoms, i.e. ataxia, nystagmus, stupor, somnolence or anorexia, ascites, weight loss of $15 \%$ in 2 days, severe pain sensations including salivation, teeth grinding, whimpering and lack of desire to move (Additional file 1: Table S1). Maternal tissue samples were taken from liver, spleen and heart. Offspring tissue samples were taken from liver, spleen, brain, heart and quadriceps femoris muscle and frozen at $-80{ }^{\circ} \mathrm{C}$ until further use. Up to $600 \mathrm{mg}$ of tissue was sampled per location. If the entire tissue or organ weighed more, samples were taken randomly from different regions. The brain was removed, and the hemispheres were separated. One hemisphere was used for qPCR; the other one was processed for histological examination.

\section{Histopathological examination}

Brains were fixed in $4 \%$ paraformaldehyde for 3 days and cut coronally into five divisions of similar thickness with a scalpel. Each brain division was embedded in paraplast and cut coronally to sections of $1 \mu \mathrm{m}$ thickness using a microtome (HM 400, Microm, Berlin, Germany). First, one section per division was stained with hematoxylin-eosin, and the number of lesions was counted in the entire section. If a lesion was found, the consecutive section was examined for the presence of microglia by immunohistochemistry using an antibody for Iba1 (2-MI004-10, Quartett, Berlin, Germany). Hematoxylin-eosin staining and immunohistochemistry were performed as described previously in [36-38]. An Olympus BX46 light microscope (Olympus, Shinjuku, Japan) equipped with an Axiocam 208 color digital camera (Zeiss, Oberkochen, Germany) and ZEN 2.6 (Zeiss, Oberkochen, Germany) imaging software was used to examine histological sections.

\section{Tissue homogenization and DNA extraction}

Tissue samples of dams and corresponding offspring were processed using NucleoMag-Tissue kit (Macherey-Nagel, Düren, Germany). The manufacturer's instructions were adjusted as follows: Tissue samples up to a maximum of $600 \mathrm{mg}$ were incubated with lysis buffer (T1) supplied with the NucleoMag-Tissue kit (Macherey-Nagel, Düren, Germany) at 1:2 ratio. Two stainless steel balls (diameter 6 mm, TIS Wälzkörpertechnologie, Gauting, Germany) 
were added, and the sample was homogenized using a TissueLyser II (Qiagen, Hilden, Germany) at $3000 \mathrm{~Hz}$ for 60 s. In a next step, Proteinase K solution provided by the NucleoMag-Tissue kit was added to the tissue buffer mixture at 1:20 ratio and the suspension incubated overnight at $56{ }^{\circ} \mathrm{C}$. All further extraction steps were carried out in a King Fisher Flex (Thermo Scientific, Waltham, MA, USA) according to the NucleoMag-Tissue kit manufacturer's instructions.

\section{Quantitative PCR}

The 529-bp repeat element, characterized by high specificity ad sensitivity for detection of $T$. gondii, was used as a target in a probe-based qPCR [39, 40]. For dams and corresponding offspring, quantitative PCR was carried out as follows: primers and probes (MWG-Biotech, Ebersberg, Germany) were applied as described previously [41] according the assay protocol "Toxo529REP PCR" (Table 1). An internal control according to [42] was used for inhibition detection as described previously [43] (Table 1). If a negative result was found to be due to PCR inhibition, the respective data were excluded from the data analysis. PCR reactions were carried out in a CFX96 cycler (Biorad Laboratories, Kabelsketal, Germany) using PerfeCTa MultiPlex qPCR ToughMix, (VWR International, Dresden, Germany) and $10 \mu \mathrm{l}$ of purified DNA. PCR conditions were as follows: 2 min at $50{ }^{\circ} \mathrm{C}$ followed by $10 \mathrm{~min}$ at $95{ }^{\circ} \mathrm{C}$ (initial denaturation). This was followed by 55 cycles, each consisting of $15 \mathrm{~s}$ at $95{ }^{\circ} \mathrm{C}$ (denaturation) and $1 \mathrm{~min}$ at $60{ }^{\circ} \mathrm{C}$ (hybridization and elongation). All qPCR results were evaluated using the CFX manager software version 1.6 (Biorad Laboratories, Kabelsketal, Germany). PCR results with a quantification cycle $(\mathrm{Cq}) \geq 40$ were considered as negative and stated as $\mathrm{Cq}=40$.

\section{Statistical analysis}

Statistical analysis was performed using Graphpad Prism 9.0.0 (GraphPad Software Inc., San Diego, CA, USA) software. Normal distribution of all parameters was tested by
Anderson-Darling test and D'Agostino and Pearson test. Since none of the data obtained by qPCR analysis and histopathological examination were normally distributed, Mann-Whitney test (for comparison of two groups) and Kruskal-Wallis test (for comparison of more than two groups) were applied for group comparison followed by Dunn's post hoc test. For regression analysis, data were analyzed by the method of least squares. For KaplanMeyer survival curves, Gehan-Breslow-Wilcoxon test was used to determine statistically significant differences between the different survival curves. $P$ values $<0.05$ were considered statistically significant. Significance levels were split further according to ${ }^{* *} P<0.01,{ }^{* * * *} P<0.001$, ****: $P<0.0001$.

\section{Results}

Fertility and pregnancy examination

A total of $83 \%$ (25/30) of dams became pregnant upon first mating. During the ultrasonographic and gynecological examination, no abnormalities were found in either the pregnant or non-pregnant animals. All remaining dams became pregnant after the second synchronization of the sexual cycle. The interval between the first mating and the start of the second cycle synchronization was at least two cycles (32 days).

\section{Impact of the infection dose and infection duration on the seroconversion}

All blood samples of the control and infection groups collected prior to infection and all blood samples of the control group collected at the end of the trial were seronegative using immunoblotting against a $T$. gondii $\mathrm{p} 30$ surface antigen (Table 2). Of the 27 dams of the infection groups, 17 (65\%) blood samples obtained at the respective end of the trial were seropositive. In infected but seronegative dams, the mean duration of infection, i.e. the time between inoculation and the respective end of the trial, was markedly shorter, i.e. 14 days, compared to that of seropositive dams, i.e. 37 days (Table 2). Moreover, logistic regression revealed a significant (Additional

Table 1 Primers, probes and their final concentrations in Toxoplasma gondii real-time PCR assay

\begin{tabular}{|c|c|c|c|c|c|}
\hline Assay & $\begin{array}{l}\text { Names of } \\
\text { primers and } \\
\text { probes }\end{array}$ & Sequences of primers and probes $5^{\prime}-3^{\prime}$ & Probe labelling & Final concentration & References \\
\hline \multirow[t]{3}{*}{ Toxo529REP PCR } & TalF & TGG TTG GGA AGC GAC GAG AG & & $800 \mathrm{nM}$ & [41] \\
\hline & TalR & CAT CAC CAC GAG GAA AGC GTC & & $800 \mathrm{nM}$ & [41] \\
\hline & TalP1_FAM & TGT CGT GCC AGC TGC ATT A & 5'-FAM, 3'-BHQ1 & $200 \mathrm{nM}$ & [41] \\
\hline \multirow[t]{3}{*}{ Internal control PCR, IC2 PCR } & EGFP1-F & GAC CAC TAC CAG CAG AAC AC & & $500 \mathrm{nM}$ & [42] \\
\hline & EGFP2-R & GAA CTC CAG CAG GAC CAT G & & $500 \mathrm{nM}$ & [42] \\
\hline & EGFP1-Hex & AGC ACC CAG TCC GCC CTG AGC A & 5'-HEX, 3'-BHQ1 & $160 \mathrm{nM}$ & [42] \\
\hline
\end{tabular}


file 2: Table S2) relationship between the probability of seroconversion and the duration of infection (Fig. 1). During the first 10 days of infection, the probability of seroconversion is $<25 \%$, rising up to $50 \%$ on day 20 of infection and reaching $90 \%$ on day 29 of infection (Fig. 1). In nine (of 10) dams, in which no seroconversion was observed, T. gondii was directly detected in at least one maternal organ using qPCR (Table 2). As T. gondii in the remaining seronegative dam was not detected by qPCR in any of the maternal and offspring organs analyzed (Table 2, no. 1), the infection of this dam was considered unsuccessful, and the dam and its offspring were excluded from the further analysis of this study.

When analyzing the effect of the dose of infection on the probability of seroconversion, no significant relationship was observed using logistic regression (data not shown; Additional file 2: Table S2).

\section{Impact of the gestational stage at infection and the infection dose on the survival rate of the dams}

All three dams of the control group survived until delivery (Figs. 2 and 3). For 11/26 (42\%) of the remaining dams of the infection groups, the trial ended prematurely. Of these 11 dams, 3 died, 2 were killed because of a critical health score $(>20 \%$ body weight loss, severe central nervous symptoms including ataxia, somnolence, stupor, and severe algesia), and 6 were killed following abortion. For the remaining 15 (58\%) dams of the infection groups, the trial ended on the day of delivery.

When the specific impact of the infection dose on the survival rate of all dams was investigated (Fig. 2), we noticed that the dose level was significantly related to the probability of survival. Administration of the highest dose, i.e. 500 oocysts, resulted in significantly (Additional file 2: Table S3) earlier loss and lower survival rate of the dams compared with administration of 10 oocysts (Fig. 2d). Moreover, administration of 100 oocysts leads to an earlier loss of dams than infection with 10 oocysts, although this difference was not statistically significant (Fig. 2d). When further taking the time of infection into account, administration of 500 oocysts on gestation day 48 resulted in a significantly (Additional file 2: Table S3) earlier loss and lower survival rate of dams than infection with 10 oocysts at the same gestational day (Fig. 2c). Again, administration of 100 oocysts on gestation day 48 leads to an earlier, although not statistically significant, loss and lower survival rate of dams than infection with 10 oocysts (Fig. 2c). A similar trend was observed for gestation days 15 and 30 with administration of 500 oocysts resulting in earlier death or euthanasia of the respective dams compared with those of lower infection doses; however, these differences were not statistically significant (Fig. 2a, b).
When analyzing the specific impact of the infection time point on the survival rate of all dams, we found a statistically significant correlation between gestational stage at infection and the probability of survival (Fig. 3). Infection in the third trimester of gestation, i.e. on gestation day 48, resulted in a significantly (Additional file 2: Table S4) earlier death or euthanasia compared with infection on day 15 of gestation (Fig. 3d). Moreover, infection on day 30 leads to an earlier loss and lower survival rate of dams than infection on day 15 of gestation, although this difference was not significant (Fig. 3d). Taking the infection dose into account, we found that infection with 500 oocysts on gestation day 48 resulted in a significantly (Additional file 2: Table S4) earlier loss and a lower survival rate of dams in comparison with infection on day 15 or 30 of gestation. Again, infection with 500 oocysts on gestation day 30 led to an earlier, although not statistically significant, loss and a lower survival rate than infection on gestation day 15 (Fig. 3c). For dams infected with 10 and 100 oocysts, no clear trend for the relationship between the infection time point and the probability of survival was observed (Fig. 3a, b).

Together, our data indicate that the probability of survival of dams infected with $T$. gondii highly depends on the dose and the gestational day at infection. Specifically, we show that higher infection doses, i.e. 500 oocysts, result in earlier loss and lower survival rate of dams than lower infection doses, i.e. 10 oocysts. Importantly, the majority of dams (78\%) infected with the highest dose, and for which the trial ended prematurely, died or were euthanized because of a critical health score (Table 2). Moreover, our data show that an infection at later time points, i.e. third trimester of gestation, led to an earlier loss of dams compared with an infection occurring at earlier time points, i.e. first trimester of gestation. Notably, the majority of dams (80\%) infected on gestation day 48 and for which the trial ended prematurely were euthanized prematurely following abortion (Table 2).

\section{Impact of the infection time point and the infection dose on the fate of the offspring}

We next investigated the fate of the offspring of each dam (Fig. 4). All three dams of the control group gave birth to asymptomatic offspring (Table 2). Of all 26 litters obtained from the infection groups, $34 \%$ of the offspring were aborted, $27 \%$ were resorbed, $23 \%$ were stillborn, and $16 \%$ were born asymptomatic (Fig. 4p). When analyzing the specific impact of the infection time point on the fate of the offspring, we found the highest survival rate for the offspring of dams infected on day 30 of gestation (Fig. 4n). Notably, infection of dams during the first trimester, i.e. on day 15 of gestation, ended lethally for $100 \%$ of the litters with the majority of offspring being resorbed 


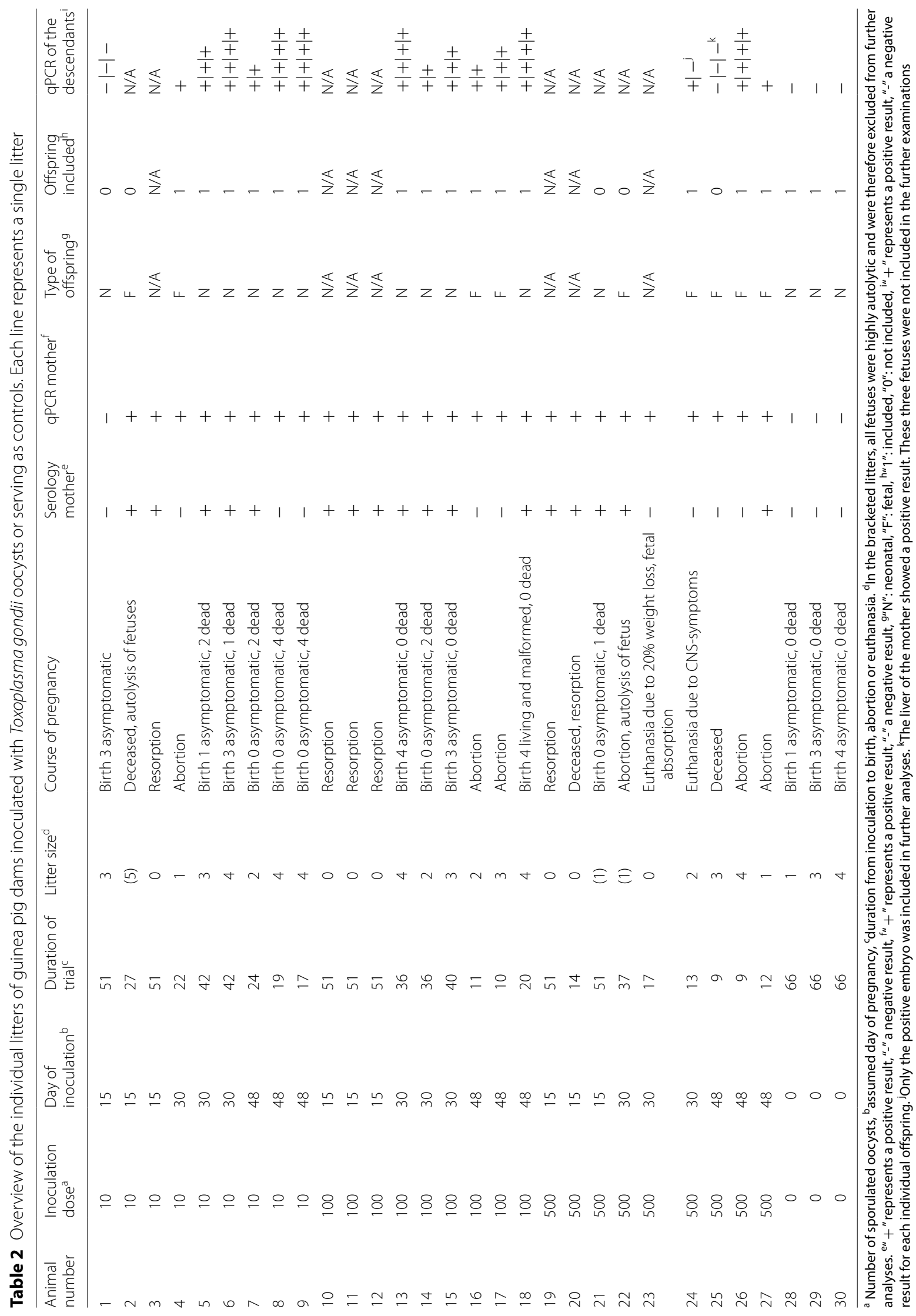




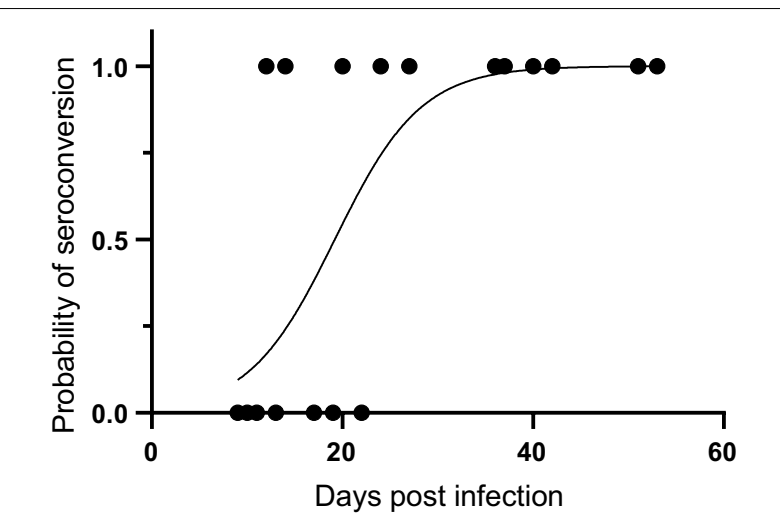

Fig. 1 Impact of the infection duration on the seroconversion. Probability of seroconversion of a dam in relation to the duration of infection. Duration of infection was defined by the time between the day of infection and the day of end of observation (day of death, euthanasia, delivery or abortion). Logistic regression analysis showed a significant correlation between both parameters analyzed $(P<$ $\left.0.001, R^{2}=0.57\right)$ during pregnancy (Fig. 4m). Moreover, infection of dams in the third trimester, i.e. on day 48 of gestation, ended lethally for the majority of litters with a very high proportion of stillbirth or abortion (Fig. 4o). Specifically, inoculation of 10 oocysts on gestation day 48 always resulted in stillbirth (Fig. 4c), whereas infection with 500 oocysts on gestation day 48 led to the abortion of all offspring (Fig. 4k).

When analyzing the specific impact of the infection dose on the fate of the offspring, we found all four possible outcomes, i.e. resorption, abortion, stillbirth and birth, to be present when dams were infected with 10 and 100 oocysts (Fig. 4d, h). The highest survival rate of the offspring was found when dams were infected with 100 oocysts (Fig. 4h). Notably, infection with the highest dose, i.e. 500 oocysts, ended lethally for $100 \%$ of the litters with the overwhelming majority of offspring being resorbed or aborted during pregnancy (Fig. 4l). Again, administration of 500 oocysts on day 15 of gestation (Fig. 4i) resulted in a higher proportion
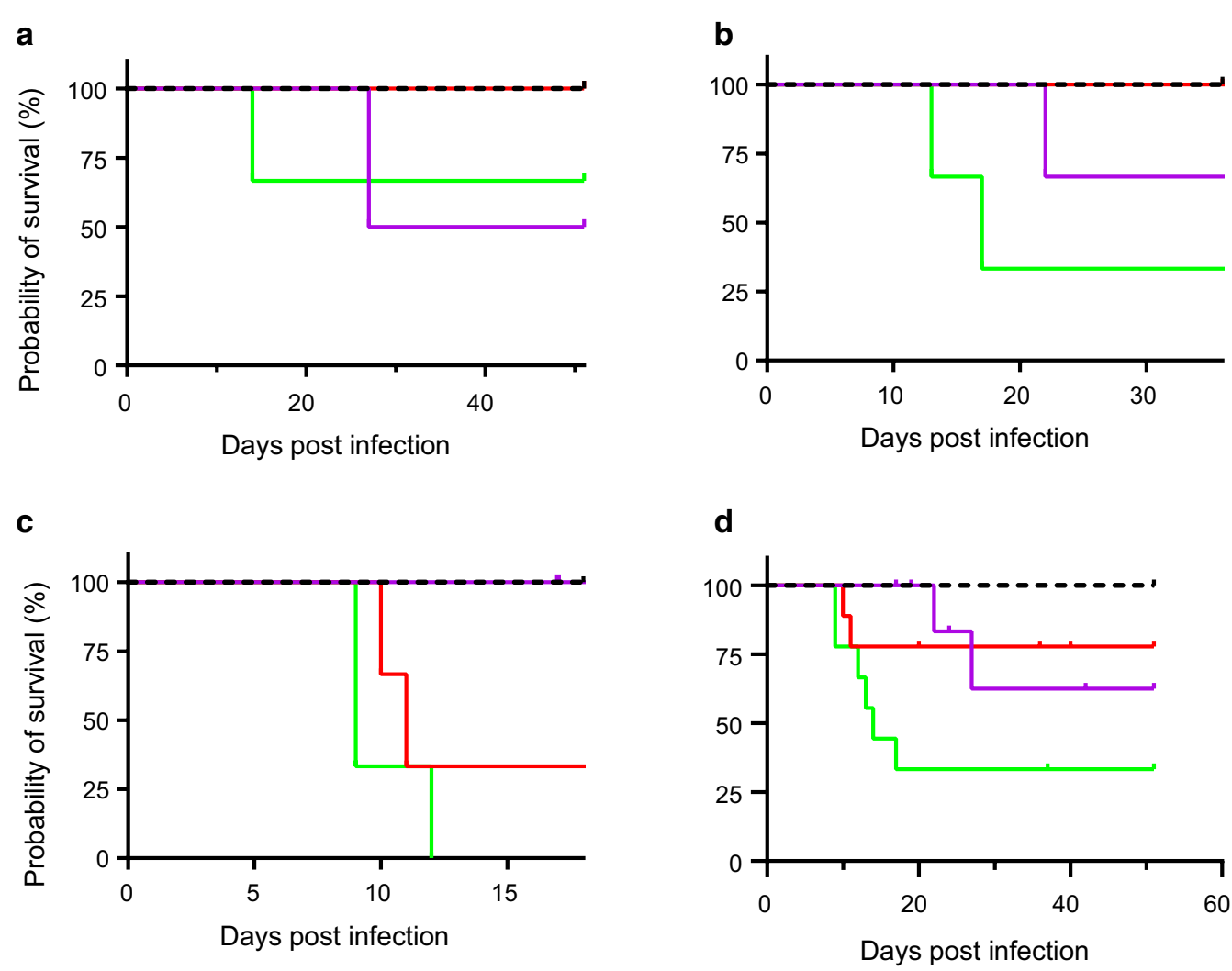

Dose: - - - - Control 10 oocysts 100 oocysts 500 oocysts

Fig. 2 Impact of the infection dose on the dam survival rate. Kaplan-Meier survival rate of dams of the control and different infection groups. Animals were infected with 10 (purple solid line), 100 (red solid line) and 500 (green solid line) sporulated T. gondii oocysts on gestation day 15 (a), 30 (b) and 48 (c). Animals of the control group (black dashed line) were administered PBS. For each dam, the day of death, euthanasia, delivery or abortion was recorded. Merged data of $(\mathbf{a}-\mathbf{c})$ are shown in (d). Statistically significant differences between groups were as follows: (c) $P<0.05$, comparing control and 500; (d) $P<0.05$, comparing 10 and 500 

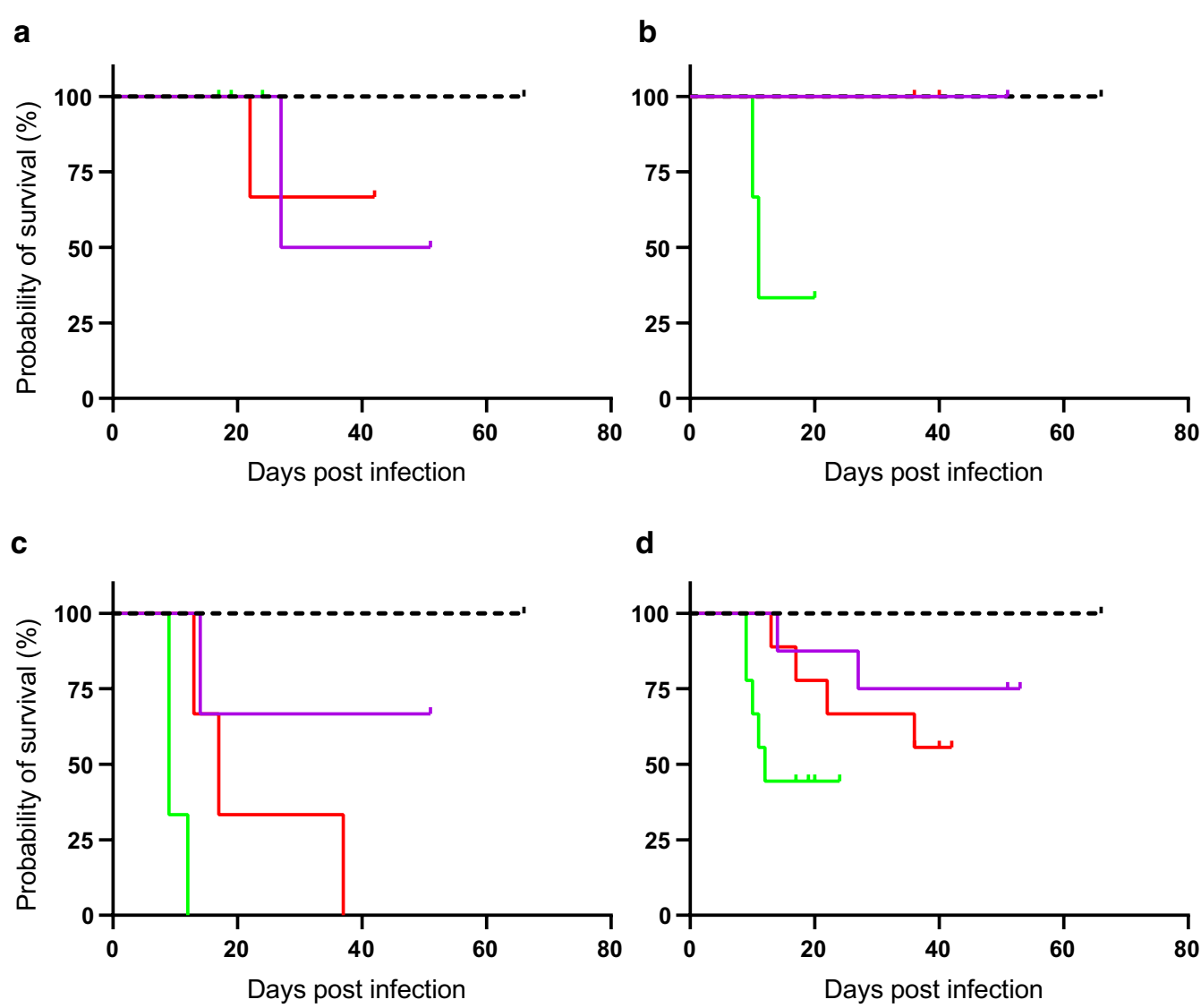

Day of infection: ---Control -15 p. c.

30 p. c. 48 p. c.

Fig. 3 Impact of the infection duration on the dam survival rate. Kaplan-Meier survival rate of dams of the control and different infection groups. Animals were infected with 10 (a), 100 (b) and 500 (c) sporulated oocysts on gestation day 15 (purple solid line), 30 (red solid line) and 48 (green solid line). Animals of the control group (black dashed line) were administered PBS. The end of the trial for a given dam was defined by death, euthanasia due to a critical health score or abortion or by euthanasia on the day of birth. Merged data of all infection days, irrespective of the day of gestation (a-c) are shown in (d). Statistically significant differences are as follows: (c) $P<0.05$, comparing control and 30; $P<0.05$, comparing control and $48 ; P<0.05$, comparing 15 and 48; $P<0.05$, comparing 30 and 48; (d) $P<0.5$, comparing 15 and 48

of fetal resorption compared with that of later gestation days (Fig. 4j, k).

Together, our data suggest that the survival rate of the offspring of dams infected with $T$. gondii depends on the dose and time point of infection. The highest survival rate of the offspring was observed when dams were infected with 100 oocysts on day 30 of gestation. However, administration of 500 oocysts, irrespective of the infection time point, and infection on day 15 of gestation, irrespective of the infection dose, ended lethally for $100 \%$ of the offspring.
Impact of infection duration and infection dose on T. gondii DNA loads in the offspring

To investigate the impact of a $T$. gondii infection on $T$. gondii DNA loads in the offspring, various offspring tissues and organs were analyzed for the presence and amount of $T$. gondii DNA by qPCR using a $T$. gondiispecific 529-bp repeat element. Of all offspring $(n=53)$ obtained from all 26 litters, 7 (13\%) delivered by three different dams infected with 10 or 500 oocysts on day 15 or 30 of gestation (Table 2, no. 2, 21 and 22) were severely autolytic and could not be used for qPCR analysis. In four (8\%) offspring of two different dams infected with 500 oocysts on day 30 or 48 of gestation (Table 2, no. 24 and 25), T. gondii DNA was not detected in any of 


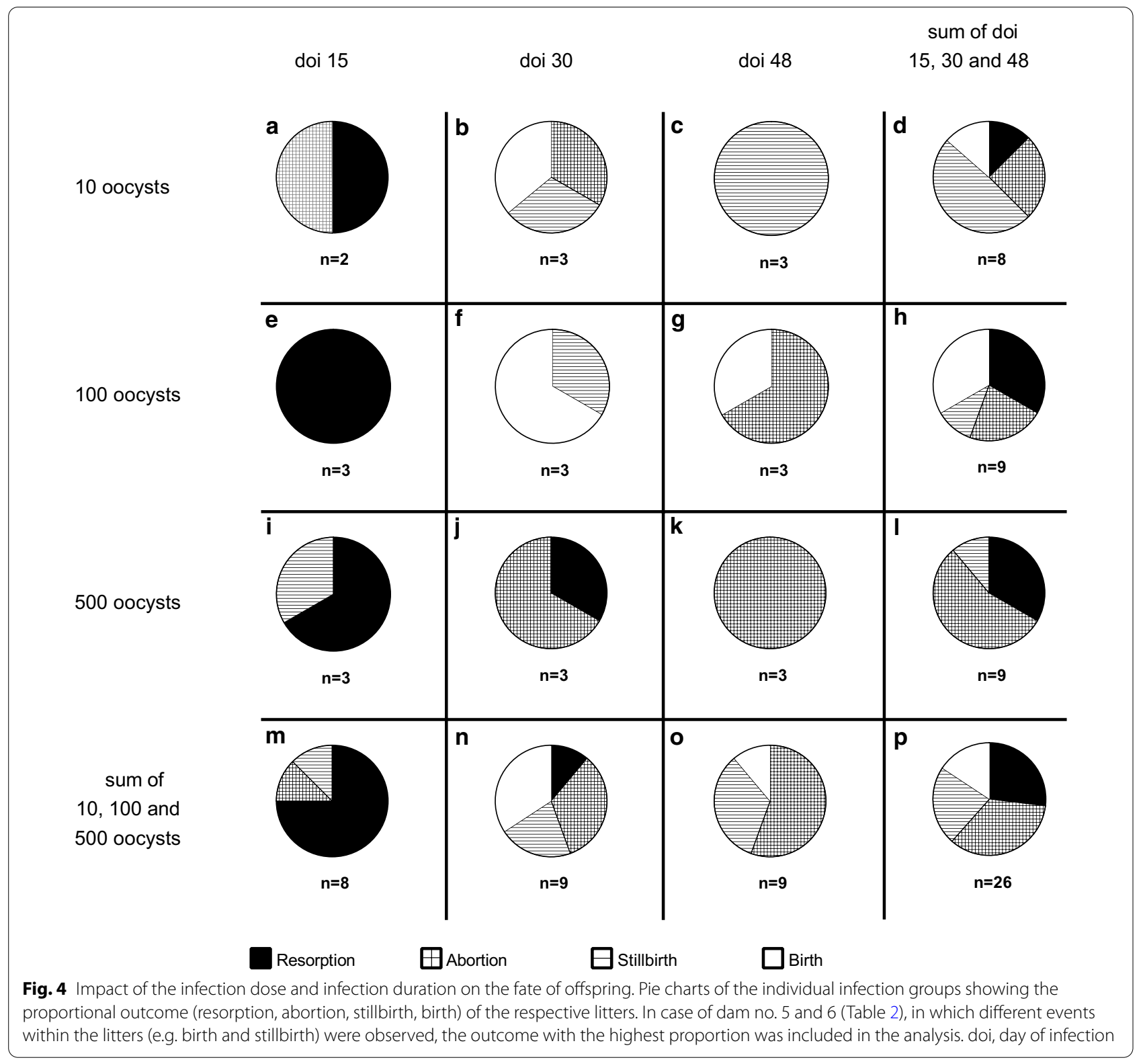

the offspring organs analyzed, i.e. brain, heart, liver, lung, spleen or femoral muscles, by qPCR (Table 2). Hence, the infection of these offspring was considered unsuccessful, and the offspring were excluded from the further analysis of this study. Given that the offspring of dams infected on day 15 of gestation, for which a T. gondii infection was verified, were either resorbed or severely autolytic (Table 2), qPCR data of this infection time point were not available, and the impact of a T. gondii infection in the first trimester of gestation on the T. gondii DNA loads in the offspring could not be analyzed. Of the remaining 42 (79\%) offspring, T. gondii DNA was detected in all brain samples (Table 3 ). Moreover, in $\geq 60 \%$ of offspring samples obtained from the heart and femoral muscles, $T$. gondii DNA was present (Table 3). Among the offspring parenchymatous organs, $T$. gondii DNA was detected in $67 \%$ of all liver samples, whereas the percentage of lung and spleen samples containing T. gondii DNA was $\leq 50 \%$ (Table 3).

We next compared the Cq values obtained by qPCR as a proxy for T. gondii DNA load between the different tissue and organ samples of all different infection groups. $\mathrm{Cq}$ values were significantly lower in the offspring brain compared with that of heart, lung and spleen (Fig. 5, Additional file 2: Table S5, Additional file 2: Table S6). No significant difference in the $\mathrm{Cq}$ values was observed when 
Table 3 Individual qPCR results for each offspring separated by organ or tissue

\begin{tabular}{|c|c|c|c|c|c|c|c|c|c|}
\hline Oocyst dose & $\begin{array}{l}\text { Day of } \\
\text { inoculation }\end{array}$ & Litter no. & Offspring & Brain & Heart & Liver & Lung & Spleen & Muscle \\
\hline 10 & 30 & 4 & A & + & - & - & - & - & - \\
\hline 10 & 30 & 5 & A & + & - & + & - & - & - \\
\hline 10 & 30 & 5 & B & + & - & - & + & $\mathbb{H}$ & + \\
\hline 10 & 30 & 5 & C & + & - & - & + & - & + \\
\hline 10 & 30 & 6 & A & + & - & - & - & - & - \\
\hline 10 & 30 & 6 & B & + & + & $\mathbb{H}$ & $\mathbb{H}$ & - & - \\
\hline 10 & 30 & 6 & C & + & + & $\mathbb{H}$ & $\mathbb{H}$ & - & - \\
\hline 10 & 30 & 6 & D & + & - & - & - & - & - \\
\hline 10 & 48 & 7 & A & + & + & + & $\mathbb{H}$ & $\mathbb{H}$ & + \\
\hline 10 & 48 & 7 & B & + & + & + & - & - & + \\
\hline 10 & 48 & 8 & A & + & + & + & $\mathbb{H}$ & + & + \\
\hline 10 & 48 & 8 & B & + & + & + & + & + & + \\
\hline 10 & 48 & 8 & C & + & + & + & 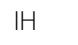 & + & + \\
\hline 10 & 48 & 8 & D & + & + & + & + & + & + \\
\hline 10 & 48 & 9 & A & + & + & + & $\mathbb{H}$ & + & + \\
\hline 10 & 48 & 9 & B & + & + & + & $\mathbb{H}$ & + & + \\
\hline 10 & 48 & 9 & $C$ & + & + & + & $\mathbb{H}$ & $\mathbb{H}$ & + \\
\hline 10 & 48 & 9 & $D$ & + & + & + & $\mathbb{H}$ & + & + \\
\hline 100 & 30 & 13 & A & + & - & - & $\mathbb{H}$ & - & - \\
\hline 100 & 30 & 13 & B & + & - & - & $\mathbb{H}$ & - & + \\
\hline 100 & 30 & 13 & $C$ & + & + & - & $\mathbb{H}$ & - & - \\
\hline 100 & 30 & 13 & $D$ & + & - & + & $\mathbb{H}$ & - & + \\
\hline 100 & 30 & 14 & A & + & - & - & + & $\mathbb{I H}$ & + \\
\hline 100 & 30 & 14 & B & + & + & + & $\mathbb{H}$ & - & + \\
\hline 100 & 30 & 15 & A & + & + & - & $\mathbb{H}$ & - & - \\
\hline 100 & 30 & 15 & B & + & + & - & - & - & + \\
\hline 100 & 30 & 15 & C & + & + & - & $\mathbb{H}$ & $\mathbb{H}$ & + \\
\hline 100 & 48 & 16 & A & + & - & + & $\mathbb{H}$ & - & $\mathbb{H}$ \\
\hline 100 & 48 & 16 & B & + & + & + & $\mathbb{H}$ & $\mathbb{H}$ & $\mathbb{H}$ \\
\hline 100 & 48 & 17 & A & + & + & + & $\mathbb{H}$ & - & $\mathbb{I H}$ \\
\hline 100 & 48 & 17 & B & + & + & + & $\mathbb{H}$ & + & $\mathbb{H}$ \\
\hline 100 & 48 & 17 & C & + & - & + & $\mathbb{H}$ & + & $\mathbb{H}$ \\
\hline 100 & 48 & 18 & A & + & + & + & $\mathbb{H}$ & - & + \\
\hline 100 & 48 & 18 & B & + & + & + & $\mathbb{H}$ & - & + \\
\hline 100 & 48 & 18 & $C$ & + & + & - & $\mathbb{H}$ & - & + \\
\hline 100 & 48 & 18 & D & + & + & + & $\mathbb{H}$ & - & + \\
\hline 500 & 30 & 24 & B & + & + & + & $\mathbb{H}$ & + & - \\
\hline 500 & 48 & 26 & A & + & + & + & $\mathbb{H}$ & + & - \\
\hline 500 & 48 & 26 & B & + & + & $\mathbb{H}$ & $\mathbb{H}$ & $\mathbb{I H}$ & - \\
\hline 500 & 48 & 26 & C & + & + & + & $\mathbb{H}$ & + & - \\
\hline 500 & 48 & 26 & $D$ & + & + & + & $\mathbb{H}$ & $\mathbb{H}$ & - \\
\hline 500 & 48 & 27 & A & + & + & + & + & + & + \\
\hline Positivity rate & & & & $100 \%$ & $71 \%$ & $67 \%$ & $50 \%$ & $38 \%$ & $62 \%$ \\
\hline
\end{tabular}

"+" represents a positive result, "-" a negative result. No result could be generated for "IH" due to PCR inhibition no. number, $I H$ inhibition 


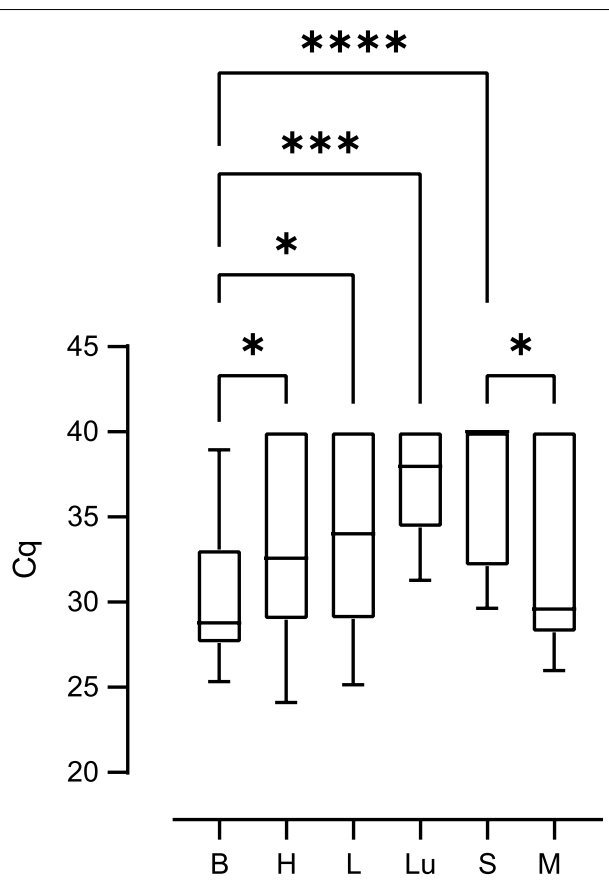

Fig. 5 Impact of T. gondii infection on Cq values for Toxoplasma gondii DNA in the offspring. Cq values for T. gondii DNA extracted from tissues and organs of offspring obtained from dams infected with 10, 100 or 500 oocysts on gestation day 15, 30 or 48 . Solid lines indicate comparison between offspring tissue or organs. ${ }^{*} P<0.05$; ${ }^{* *} P<0.01$; ${ }^{* * *} P<0.0001$. B brain, $H$ heart, $L$ liver, Lu lung, $S$ spleen, $M$ femoral muscles

offspring heart, liver and lung were compared (Fig. 5). Since the infection duration varies greatly between the different infection groups, even when dams were infected on the same gestational day, we focused our analysis on the impact of the actual duration dams were infected, irrespective of the infection time point, on the amount of T. gondii DNA in the offspring tissue and organ samples (Fig. 6). For this, the infection duration of dams was subdivided into three different groups: $0-16,17-33$ and 34-51 days.

When comparing $\mathrm{Cq}$ values between different tissues and organs within the same group of duration of infection (Fig. 6), our data show that T. gondii infection of dams for a duration of 34-51 days results in significantly lower $\mathrm{Cq}$ values in the offspring brain compared with those of heart, liver, lung, spleen and femoral muscles (Fig. 6c, Additional file 2: Table S5, Additional file 2: Table S7). Similarly, when dams were infected for a shorter duration, i.e. 17-33 days, Cq values in the offspring brain were significantly lower compared with those of spleen; however, they did not differ markedly from values in the heart, femoral muscles, lung and liver for the same infection duration of the dam (Fig. 6b, Additional file 2: Table S5, Additional file 2: Table S7). No significant differences between the Cq values of all offspring tissues and organs analyzed were observed when dams were infected for 0-16 days (Fig. 6a).

When comparing Cq values of the same tissue or organ between different groups of infection durations, $\mathrm{Cq}$ values in the offspring heart, liver, spleen and femoral muscles were significantly higher when dams were infected for 34-51 days (Fig. 6c, Additional file 2: Table S5, Additional file 2: Table S7) compared to those infected for a shorter duration, i.e. 17-33 days, or in case of the spleen 0-16 days (Fig. 6a, b). In contrast, median Cq values were found to decrease in the offspring brain with increasing infection duration of a dam. Specifically, Cq values in the offspring brain were significantly lower when dams were infected for 34-51 or 17-33 days (Fig. 6b, c, Additional file 2: Table S5, Additional file 2: Table S7) than in the offspring brain of dams infected for $0-16$ days (Fig. 6a), respectively.

Together, our data show that the duration of T. gondii infection impacts on the amount of T. gondii DNA in the offspring with longer infection durations of pregnant dams, i.e. 34-51 days, relating to lower $\mathrm{Cq}$ values, and thus obviously higher $T$. gondii DNA levels, in the offspring brain and to higher Cq values, and thus obviously lower $T$. gondii DNA levels in most of the offspring abdominal organs examined. This observation is further supported by the estimation of the relationship between the $\mathrm{Cq}$ value obtained in the offspring brain and liver and infection durations of dams as analyzed by regression analysis (Fig. 7, Additional file 2: Table S5, Additional file 2: Table S8). In particular, it shows that Cq values and infection duration are significantly negatively correlated (Fig. 7a). In contrast, in the offspring liver, Cq value and infection durations are significantly positively correlated (Fig. 7b).

We next analyzed the impact of the infection dose on the amount of T. gondii DNA in the offspring tissue and organ samples (Fig. 8). When comparing Cq values between different tissues and organs of offspring obtained from dams infected with the same infection dose, our data show that administration of 10 and 100 oocysts results in significantly lower Cq values in the offspring brain compared with those of spleen, lung or liver (Fig. 8a, b, Additional file 2: Table S5, Additional file 2: Table S9). No significant differences, except for spleen and femoral muscles infected with 100 oocysts, were observed when different tissues and organs were compared following an administration of 100 or 500 oocysts, respectively (Fig. 8b, c).

When comparing $\mathrm{Cq}$ values of the same tissue or organ between different infection doses, $\mathrm{Cq}$ values in the offspring brain were significantly higher when dams were infected with 500 oocysts (Fig. 8c, Additional 
a

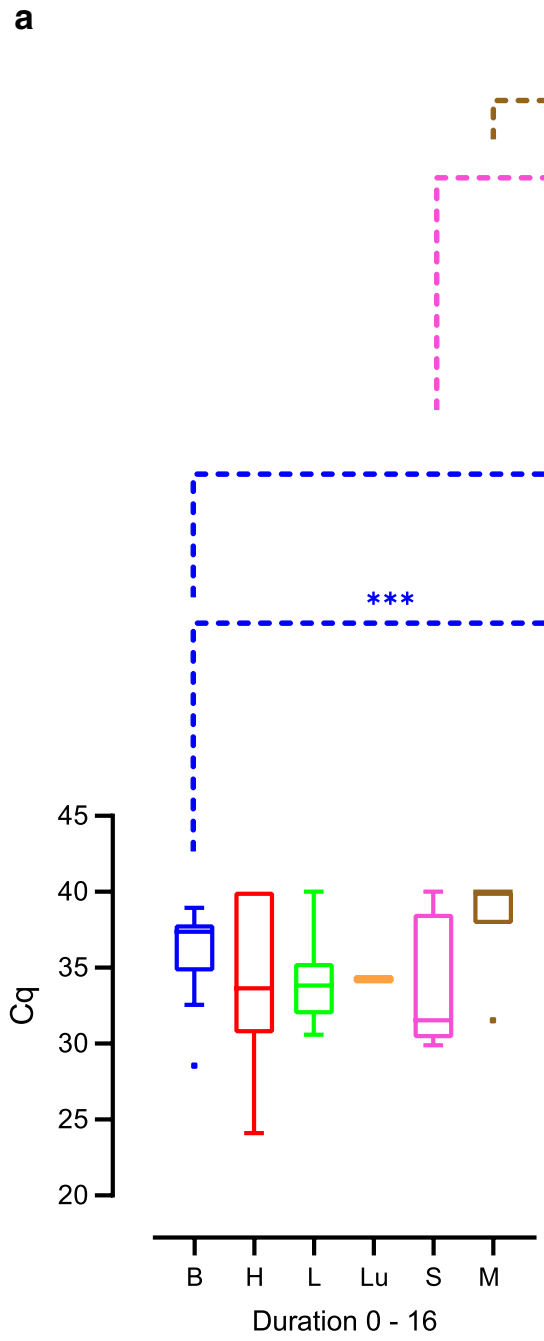

b

C
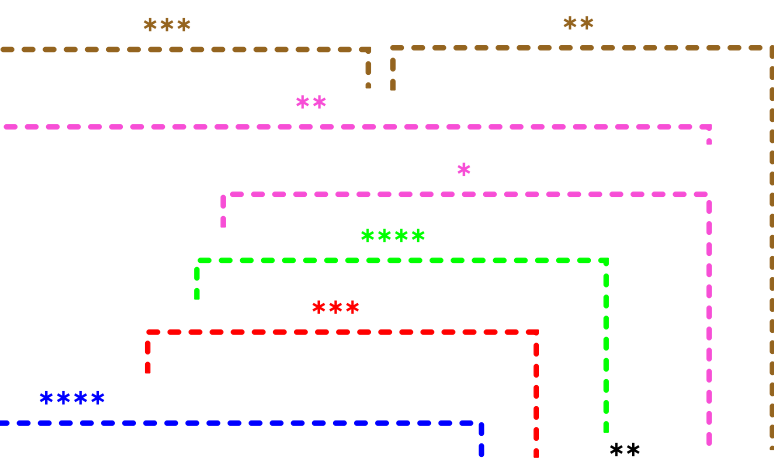

-

$* * * *$
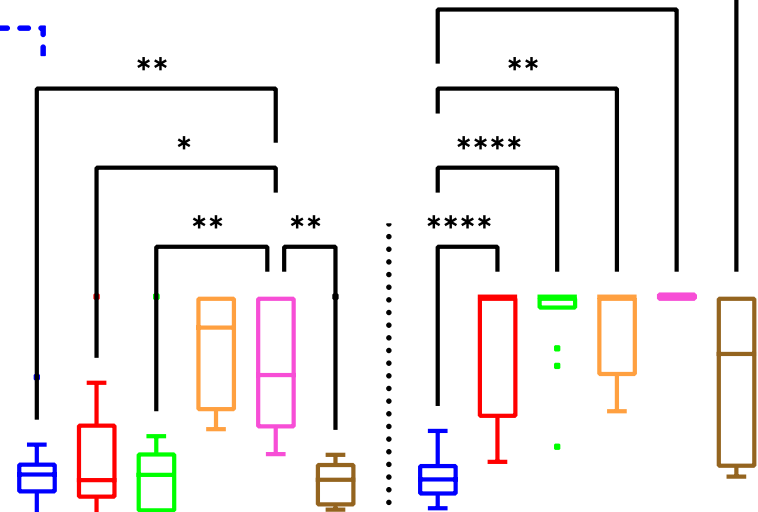

Fig. 6 Impact of the infection duration on Cq values for Toxoplasma gondii DNA in the offspring. Cq values for T. gondii DNA extracted from tissues and organs of offspring obtained from dams infected for different durations. Solid lines indicate comparison of offspring organs obtained from dams infected for the same inoculation duration. Dashed lines indicate comparison of the same organ between different offspring survival times. ${ }^{*} P<0.05 ;{ }^{* *} P<0.01 ;{ }^{* *} P<0.001 ;{ }^{* * *} P<0.0001$. B brain, $H$ heart, $L$ liver, Lu lung, $S$ spleen, $M$ femoral muscles

file 2: Table S5, Additional file 2: Table S9) compared with those infected with lower infection doses, i.e. 10 and 100 oocysts (Fig. 8a, b), and Cq values in the offspring brain were significantly higher when dams were infected with 100 oocysts (Fig. 8b) compared with those infected with 10 oocysts (Fig. 8a). Moreover, significantly higher $\mathrm{Cq}$ values were observed in the offspring femoral muscles following an infection with 500 oocysts (Fig. 8c) and liver following an infection with 100 oocysts (Fig. 8c) compared with those infected with a lower infection dose, i.e. 10 oocysts (Fig. 8a). No significant differences between different infection doses were observed when all other organs were compared (Fig. 8).
When comparing $\mathrm{Cq}$ values of various tissues and organs of offspring obtained from dams infected for the same infection duration, i.e. 34-51 days, no significant differences were observed when 10 and 100 oocysts were administered (Fig. 9, Additional file 2: Table S5, Additional file 2: Table S10). This indicates that the T. gondii infection dose has less impact on the amount of $T$. gondii DNA level in the offspring.

Last, we investigated whether the amount of T. gondii DNA in the guinea pig offspring corresponded to its survival and compared $\mathrm{Cq}$ values of various tissue and organs of stillborn offspring and those born asymptomatic, all obtained from dams infected for the same infection duration, i.e. 34-51 days. This reveals that Cq 
a

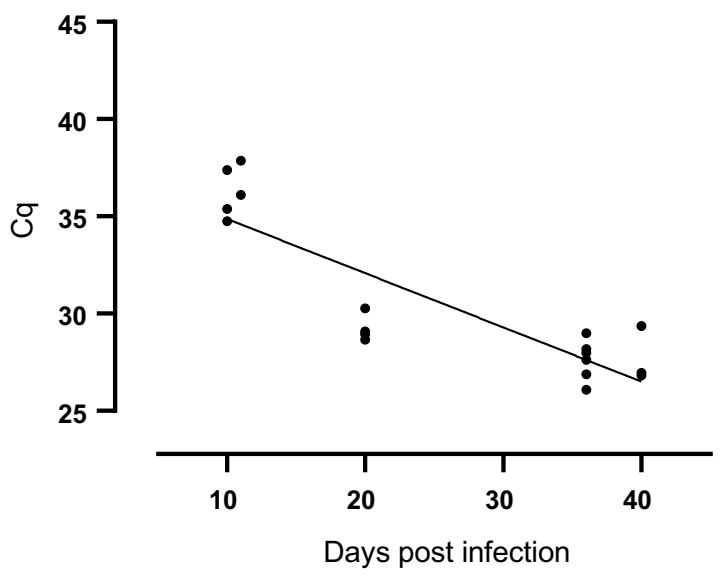

b

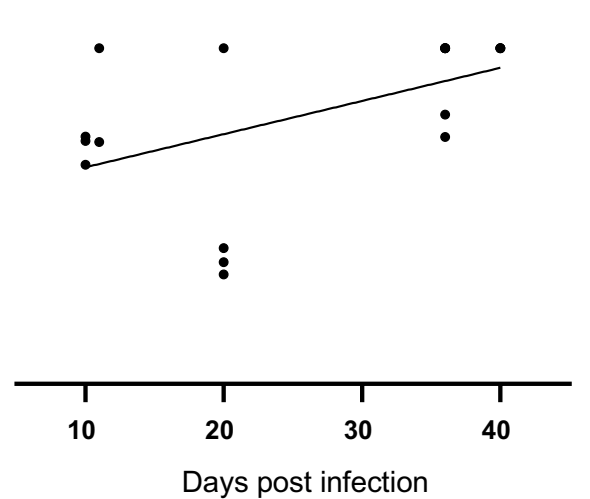

Fig. 7 Relationship between duration of infection and Cq values for Toxoplasma gondii DNA in the offspring. Cq values of offspring brain (a) and liver (b) obtained from dams infected with 100 oocysts as determined by T. gondii qPCR. For offspring brain, the regression was characterized by $P<0.0001, R^{2}=0.7464$ and $f(x)=-0.2786 x+37.65$. For offspring liver, the regression was characterized by $P<0.05, R^{2}=0.2556$ and $f$ $(x)=0.1702 x+32,20$. Data were analyzed using the least squares method

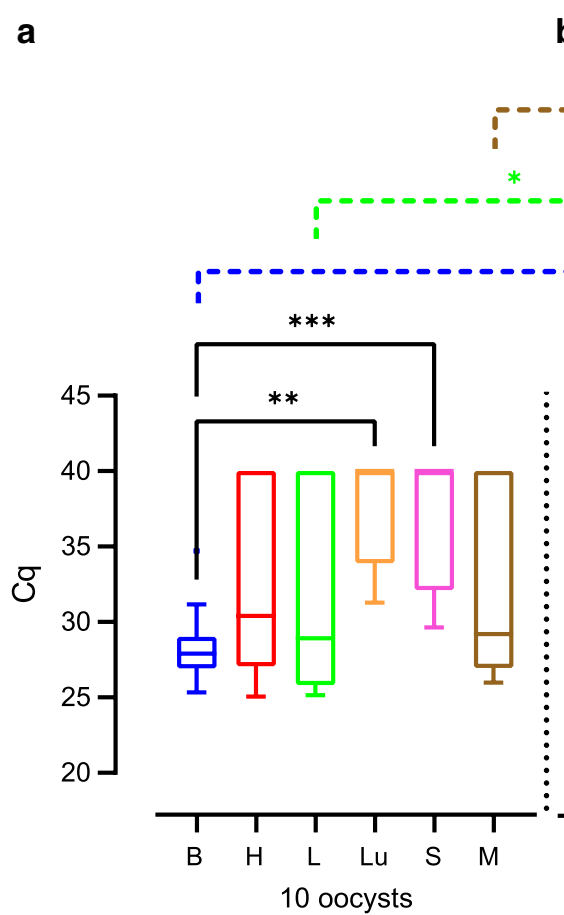

b

C

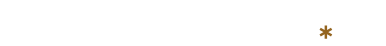

\section{(1)}




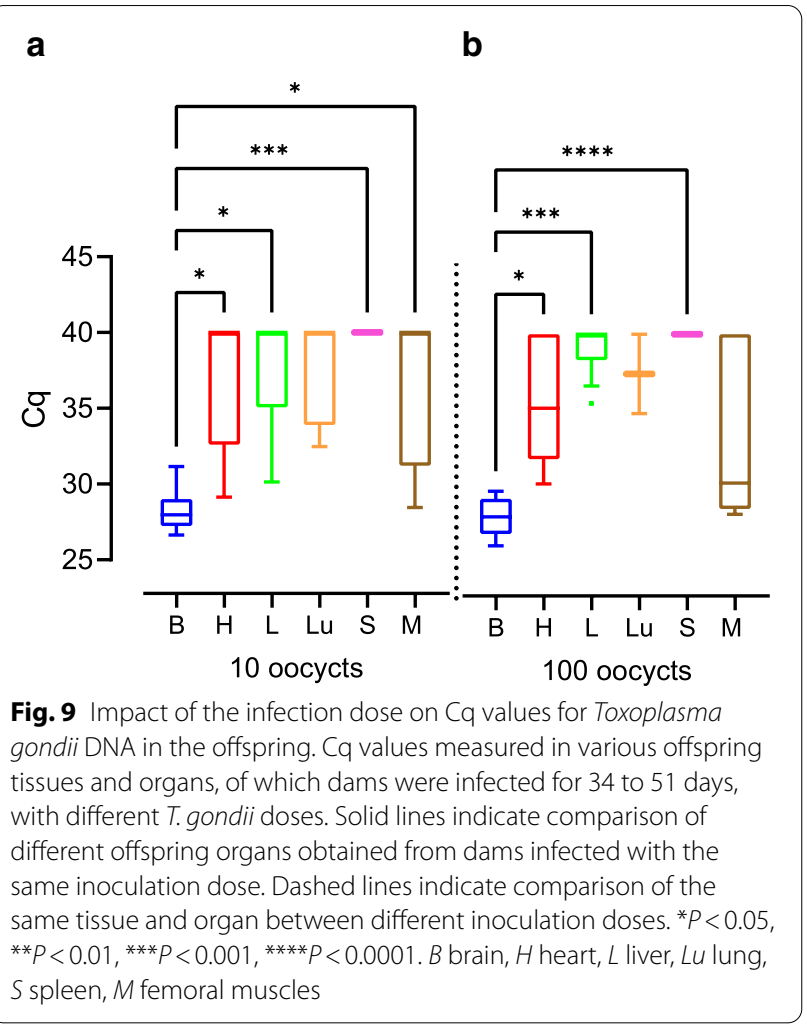

values of offspring brain, liver, heart, lung, spleen and femoral muscle did not differ significantly between stillborn offspring and those born asymptomatic (Additional file 2: Table S11, Additional file 3: Figure S1), thus indicating that the amount of T. gondii DNA in the guinea pig offspring might not corresponds to survival.

\section{Impact of $T$. gondii infection on the integrity of the offspring brain}

In a next step, we assessed the impact of $T$. gondii infection on the integrity of the offspring brain by pathohistological examination. For this, the brain of all offspring was cut coronally into five divisions of similar thickness and one section per brain division was stained with hematoxylin-eosin and an antibody for Iba1, a marker of microglia. In all offspring of the control group, no pathological changes including necrotizing necrosis lesions were detected in the brain. In contrast, in the offspring of the infection groups, focal or multifocal microgliosis and/or necrotizing encephalitis were present in numerous brains (Fig. 10). Moreover, within the center of some lesions, intracellular protozoal structures were detectable in intracellular vacuoles (Fig. 10b). A total of 50 microglia-associated lesions were quantified in the brain sections of all offspring analyzed. When comparing the number of lesions per offspring brain between dams infected for different durations, our data show that the number of lesions observed in the offspring brain of dams

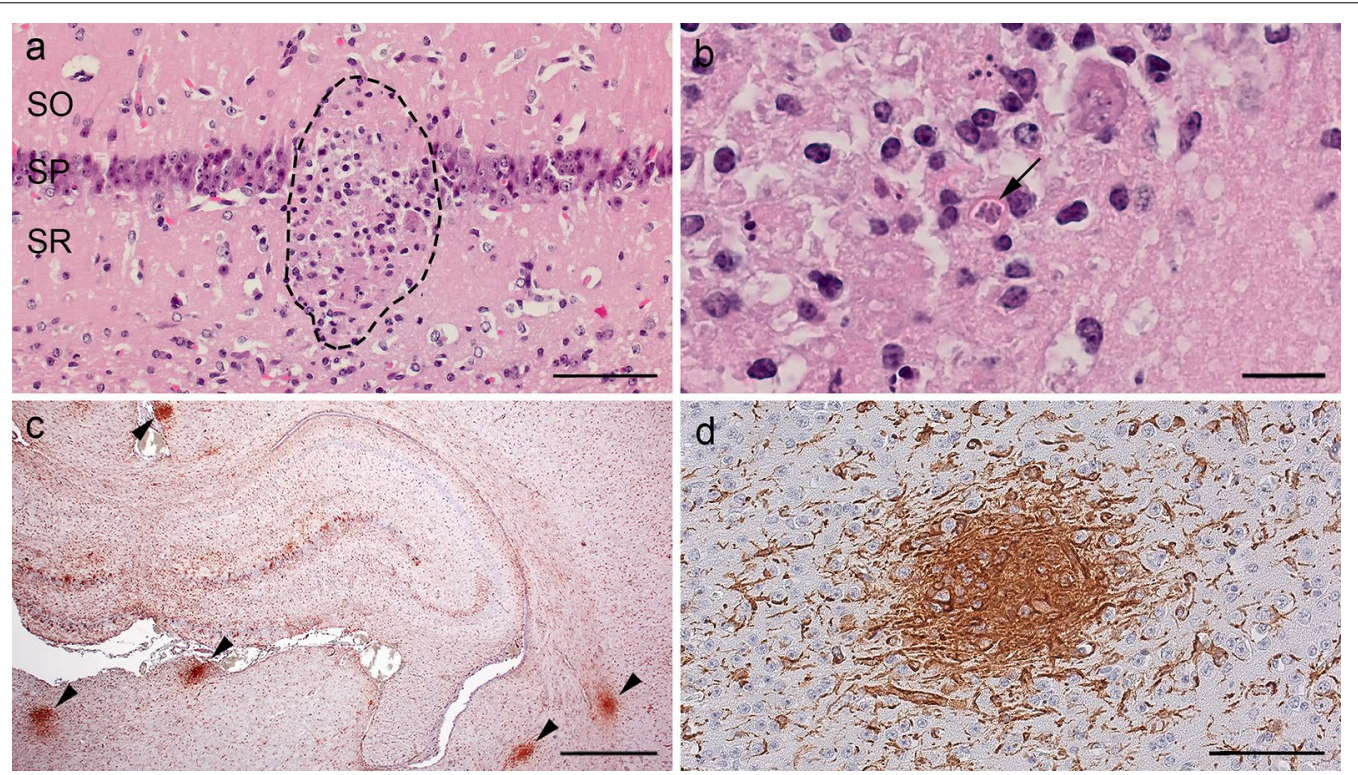

Fig. 10 Histological examination of the offspring brain infected with Toxoplasma gondii. a A focal necrosis with microgliosis in the hippocampus stained with hematoxylin-eosin. Dashed lines indicate delineation of the focus. SO, stratum oriens; SP, stratum pyramidale; SR, stratum radiatum, scale bar $=50 \mu \mathrm{m}$. b A subset of $\mathbf{a}$ in higher magnification, scale bar $=10 \mu \mathrm{m}$. The arrow indicates intracytoplasmic tachyzoites. c An overview image of an IBA 1 immunohistochemical stain in the hippocampal region. Arrowheads indicate the IBA 1-positive foci, scale bar $=500 \mu \mathrm{m}$. $\mathbf{d}$ A subset of $\mathbf{c}$ in higher magnification (scale bar $=50 \mu \mathrm{m}$ ) 

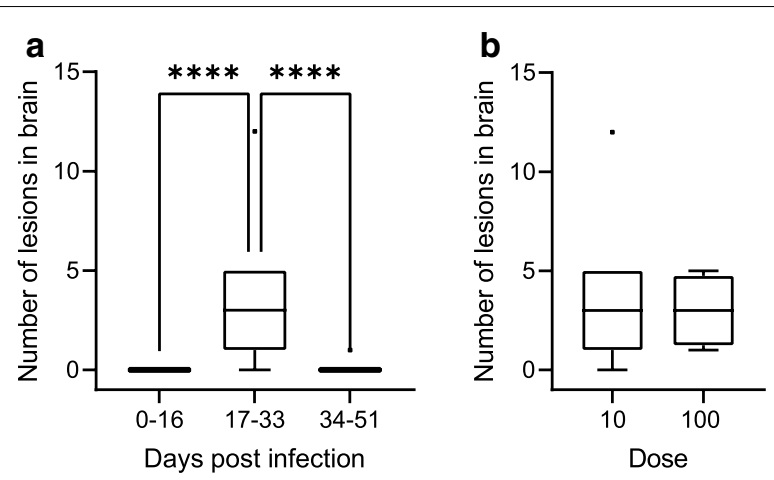

Fig. 11 Impact of the infection duration and infection dose on the integrity of offspring brain. a The number of lesions detected per offspring brain in relation to the duration dams were infected ${ }^{* * * *} P<0.0001$ ). $\mathbf{b}$ The number of lesions per offspring brain, of which dams were infected for 17 to 33 days, in relation to the infection dose. $\mathbf{a}, \mathbf{b}$ Number of lesions was counted in the five brain sections of each brain. For details, see Methods

infected for 17-33 days was significantly higher than that of those obtained from dams infected for $0-16$ and 34-51 days (Fig. 11a, Additional file 2: Table S12, Additional file 2: Table S13). When comparing the number of lesions per offspring brain between dams inoculated with different doses within the same duration of infection, i.e. 17-33 days, no significant differences could be observed (Fig. 11b, Additional file 2: Table S12, Additional file 2: Table S13).

Together, our data show that $T$. gondii infection affects the integrity of the offspring brain. Specifically, T. gondii infection of dams for 17-33 days results in a high abundance of nodular microgliosis in the offspring brain, whereas the infection dose seems to have little impact on the abundance of microglia-associated lesions in the offspring brain.

\section{Discussion}

This project provides extensive contributions to establishing the guinea pig as a suitable animal model for (human) congenital toxoplasmosis. Since all dams became pregnant at least upon second mating and no abnormalities were found in either the pregnant or nonpregnant animals, we conclude that fertility and reproductive problems are unlikely to have interfered with the $T$. gondii infection results. By oral administration of T. gondii oocysts, we chose a natural route of infection for the guinea pig, because as an herbivorous animal it becomes infected almost exclusively via oocyst-contaminated food or water. Oocyst infection also plays an important role in humans, since up to $43-78 \%$ of human T. gondii infections are considered to be transmitted via oocyst-contaminated food or water $[44,45]$. As this is the first study using the ME49 strain for congenital infections in guinea pigs, T. gondii oocyst doses were chosen based on previous studies using oocysts of ME49 strain for congenital infection in mice and adapted to account for the (approximately 20x) higher guinea pig body weight [46].

We show that the probability of seroconversion in $T$. gondii-infected pregnant guinea pigs strongly depends on the duration a dam is infected with its probability exceeding $50 \%$ after day 20 of infection. This is in line with previous studies in guinea pigs and other mammals showing seroconversion to usually occur between 9 and 21 days post-infection [32, 46-48]. Given that in almost all seronegative dams $T$. gondii was directly detected in at least one maternal organ using qPCR, it can be assumed that the time between infection and sampling in those dams was simply not long enough for specific antibodies to become detectable in the blood. In addition, our data indicate the dose of infection seems to have little impact on the probability of seroconversion in T. gondii-infected pregnant guinea pigs, which also agrees with previous findings in mice [46].

Similar to findings in guinea pigs and other mammalian species $[21,22,46,48]$, we found that the survival rate of T. gondii-infected dams strongly differs depending on the dose of infection. In general, it was found that increasing oocyst doses caused earlier offspring losses and lower survival rates of pregnant dams. Specifically, administration of the highest infection dose, i.e. 500 oocysts, results in a significantly earlier loss and lower survival rate of dams than infection with lower infection doses, i.e. 10 oocysts. In this regard, a potential relationship between the pregnancy and a low survival rate of dams cannot be excluded. As this is the first study using oocysts of ME49 strain for infection in guinea pigs, further studies including $T$. gondii infections of non-pregnant animals are needed to determine whether a low survival rate, especially after administration of 500 oocysts, is related to pregnancy or rather a general phenomenon in guinea pigs.

Moreover, we show that infection with 500 oocysts always ended lethally for the respective litters, with > $50 \%$ of offspring being aborted. A similar dose-dependent effect on the fate of the offspring has been previously observed in guinea pigs and other mammals including mice showing that higher $T$. gondii doses generally result in a smaller litter size $[21,46,48]$. Additionally, the fate of the offspring seems to depend on the gestational stage at which the dam is infected. In particular, a T. gondii infection of dams during the first trimester, i.e. on day 15 of gestation, ended lethally for all litters with most offspring being resorbed during pregnancy, as has been shown in humans $[19,49]$. 
Intriguingly, infection of dams in the third trimester, i.e. on day 48 of gestation, ended lethally for most litters with a very high proportion of offspring stillbirth or abortion. Similarly, a previous study in guinea pigs showed that $T$. gondii infection at the end of the second trimester of gestation leads to significantly higher offspring losses compared to infection in the first trimester of gestation [21]. However, our findings contrast with findings in humans, in which infection during the third trimester of gestation often remains asymptomatic for the fetus $[49,50]$. One possible scenario for this difference might be that T. gondii infiltrates and disrupts the placenta more severely in guinea pigs compared to humans upon an infection in the third trimester of gestation. Given that the placental tissue was consumed by the dams immediately after abortion, an examination of the placental tissue was not possible in this study. We therefore recommend this analysis to be part of further studies.

Furthermore, we show that the duration a pregnant dam is infected strongly impacts on the amount of $T$. gondii DNA in the guinea pig offspring. Specifically, a $T$. gondii infection of dams for $<17$ days appears to result in similar T. gondii DNA loads in various offspring organs and tissues, namely brain, liver, spleen, heart, lung and femoral muscles. However, an infection of pregnant dams for $>34$ days seems to correspond to higher T. gondii DNA levels in the offspring brain but lower T. gondii DNA levels in all body cavity organs examined. This indicates that $T$. gondii initially replicates in various offspring tissues and organs during the acute stage of infection after which it tends to disappear from most of the body cavity organs to accumulate in the brain. Moreover, our findings support the notion that the brain is considered the preferred host organ of the permanent stages of the parasite in mammals including guinea pig and human $[22,46,51,52]$.

Interestingly, it was found that administration of high T. gondii infection doses, i.e. 500 oocysts, might result in lower $T$. gondii DNA levels in the offspring brain compared to low doses, i.e. 10 oocysts. Considering that higher infection doses significantly reduce offspring survival time, lower $T$. gondii DNA levels in the offspring organ, i.e. the brain, upon administration of high infection doses might reflect the fact that the duration of infection was simply not long enough for the parasite to accumulate in various offspring organs. Indeed, for all dams, which were administered 500 oocysts the trial ended within the first 16 days after infection. Hence, when $\mathrm{Cq}$ values of various tissue and organs of offspring obtained from dams infected were compared for the same infection duration, no significant differences were observed between the different oocyst infection doses administered. Thus, our data suggest that the $T$. gondii infection dose seems to have little, if any, influence on the amount of T. gondii DNA in the guinea pig offspring.

The duration a pregnant dam is infected also affects the integrity of the offspring brain. In particular, infection of dams for 17-33 days results in significantly increased nodular microgliosis in the offspring brain compared to shorter periods of infection, thus demonstrating that focal cell lesions caused by tachyzoites trigger a microglia immune response in the acute phase of $T$. gondii infection [16]. Intriguingly, the abundance of microgliaassociated lesions was significantly lower in the offspring brain when dams were infected for $>34$ days compared to 17-33 days. This potentially reflects the fact that, once the infection is controlled by the immune system, tachyzoites transform into bradyzoites, which are able to escape the host immune response [16, 53]. Again, the infection dose seems to have little impact on the abundance of microglia-associated lesions and thus the integrity of the offspring brain.

Taken together, our data show that the ME49 strain of T. gondii is vertically transmitted in the guinea pig and infects the unborn offspring, thus demonstrating its suitability for studying congenital toxoplasmosis. Based on our data, infection of pregnant dams with 10-100 T. gondii oocysts in the second trimester of gestation is suitable for investigating the course of $T$. gondii infection in the fetus and postnatal animal, specifically the T. gondii-associated pathological changes, underlying pathomechanisms and immune responses in various offspring tissues and organs including the brain. Moreover, infection of pregnant guinea pigs in the first trimester of gestation is particularly suited to investigate the pathogenesis of fetal resorption and to examine treatment options to prevent the dramatic outcome of toxoplasmosis during the early stage of infection in pregnant women. Further studies including varying $T$. gondii doses and a larger number of animals are needed to obtain detailed data on the impact of a congenital T. gondii infection on the survival of dams and their offspring as well as on parasite loads in tissues and organs of prenatally infected offspring.

\section{Conclusions}

This study demonstrates that the ME49 strain of $T$. gondii is suitable for studying congenital toxoplasmosis. Specifically, it reveals that the T. gondii infection dose and the gestational stage at infection markedly influence the survival rate of dams and the fate of the offspring. Moreover, the duration of infection substantially impacts the seroconversion rate of dams, the $T$. gondii DNA loads in the offspring and the integrity of offspring brain. Together, this work contributes significantly to establishing the guinea pig as a suitable animal 
model for human congenital toxoplasmosis, which can be used to solve scientific questions of high medical significance, i.e. regarding the pathogenesis, immunological responses, efficacy of vaccines and drugs as well as the establishment of practice-relevant diagnostic markers of congenital $T$. gondii infection in humans.

\section{Abbreviations}

B: Brain; bp: Base pair; bw: Body weight; Cq: Quantification cycle; DNA: Deoxyribonucleic acid; doi: Day of infection; F: Fetal; H: Heart; IC: Internal control; IH: Inhibition; L: Liver; Lu: Lung; M: Femoral muscles; N: Neonatal; PBS: Phosphatebuffered saline; p. c.: Post-conceptionem; PCR: Real-time quantitative polymerase chain reaction; $\mathrm{QPCR}$ : Real-time quantitative polymerase chain reaction; $\mathrm{S}$ : Spleen; SB: Stillbirth; BA: Born alive; SO: Stratum oriens of hippocampus; SO: Stratum pyramidale of hippocampus; SR: Stratum radiatum of hippocampus; T. gondii: Toxoplasma gondii; TgREP-529: 529-Base pair repetitive element of T. gondii.

\section{Supplementary Information}

The online version contains supplementary material available at https://doi. org/10.1186/s13071-021-04890-4.

Additional file 1: Table S1. Scoring system used to evaluate the clinical condition of guinea pigs.

Additional file 2: Table S2. Logistic regressions of the probability of seroconversion depending on the duration of infection and the infectious dose as shown in Fig. 1. Table S3. Individual results of the multiple GehanBreslow-Wilcoxon tests as shown in Fig. 2. Table S4. Individual results of the multiple Gehan-Breslow-Wilcoxon tests as shown in Fig. 3. Table S5. Cq values of individual organs of each offspring. Table S6. Individual results of the Kruskal-Wallis tests as shown in Fig. 5. Table S7. Individual results of the Kruskal-Wallis tests as shown in Fig. 6. Table S8. Individual results of the simple linear regressions of brains and hearts as shown in Fig. 7. Table S9. Individual results of the Kruskal-Wallis tests as shown in Fig. 8. Table S10. Individual results of the Kruskal-Wallis tests as shown in Fig. 9. Table S11. Individual results of the Mann-Whitney tests as shown in Additional file 3: Figure S1. Table S12. Number of histopathologically determined lesions in the brain of each offspring. Table S13. Individual results of the Kruskal-Wallis and Mann-Whitney tests as shown in Fig. 11.

Additional file 3: Figure S1. Impact of the Cq value on the fate of offspring. Cq values measured in various offspring tissues and organs, of which dams were infected for 34 to 51 days, with different $T$. gondii doses. No significant differences were found between the stillborn offspring and the offspring born asymptomatic. SB stillbirth, BA born alive.

\section{Acknowledgements}

We thank Hilke Gräfe, Gabriele Lindner, Elfi Quente, Kevin Richter, Yasmina Schmitt, Andrea Bärwald, Alrik-Markis Kunisch, Mareen Tuschy and Susanne Zahnow for their technical support as well as Katharina Rauhe and Katrin Balzejak for their support in oocyst collection. We gratefully thank Hans-Jürgen Raqué (Veterinary Practice Raqué, Leipzig) for providing the ultrasonography equipment. We acknowledge support from Leipzig University for Open Access Publishing.

\section{Authors' contributions}

SAF conceived. SAF and AD designed and supervised study TG, BB, GS, PM, $L R$ and $R U$ performed research. CS contributed materials. TG analyzed data. TG, ZRS, GS, PM, JS, RU, AD and SF discussed data. TG, GS, AD and SAF wrote the paper. ZRS, CS, RU reviewed and edited the paper. All authors read and approved the manuscript.

\section{Funding}

Open Access funding enabled and organized by Projekt DEAL. This work was supported by the National Research Platform for Zoonoses/Federal Ministry of
Education and Research (01KI1801), and the Research Academy Leipzig and the Vice-Rector for Research and Young Academics (Pre-Doc-Award conferred to Thomas Grochow and Simone A. Fietz), University of Leipzig, Germany.

\section{Availability of data and materials}

The data generated or analyzed during this study are included in this published article or can be obtained from the corresponding author on request.

\section{Declarations}

\section{Ethics approval and consent to participate}

All animal experiments were performed in accordance with German animal welfare legislation. The guinea pig study was approved by the Landesdirektion Sachsen (TVV 45/17, DD24.1-5131./390/47) and infection of cats to provide oocyst infection material was permitted by the ethics commission of the Animal Care and Use Committee of the German Lower Saxony State Office for Consumer Protection and Food Safety (Niedersaechsisches Landesamt für Verbraucherschutz und Lebensmittelsicherheit; reference number 33.19-42502-05-17A206).

\section{Consent for publication}

Not applicable.

\section{Competing interests}

The authors declare that they have no competing interests.

\section{Author details}

${ }^{1}$ Institute of Veterinary Anatomy, Histology and Embryology, Faculty of Veterinary Medicine, University of Leipzig, Leipzig, Germany. ${ }^{2}$ Institute of Parasitology, Faculty of Veterinary Medicine, University of Leipzig, Leipzig, Germany. ${ }^{3}$ National Reference Laboratory for Toxoplasmosis, Institute of Epidemiology, Friedrich-Loeffler-Institut, Federal Research Institute for Animal Health, Greifswald-Insel Riems, Germany. ${ }^{4}$ Institute for Parasitology, Centre for Infection Medicine, University of Veterinary Medicine Hannover, Hannover, Germany. ${ }^{5}$ Veterinary Practice Raqué, Leipzig, Germany. ${ }^{6}$ Institute of Veterinary Pathology, Faculty of Veterinary Medicine, University of Leipzig, Leipzig, Germany.

Received: 4 May 2021 Accepted: 23 July 2021

Published online: 06 August 2021

\section{References}

1. Blader I, Coleman B, Chen C-T, Gubbels M-J. The lytic cycle of Toxoplasma gondii: 15 years later. Annu Rev Microbiol. 2015;69:463-85.

2. Opsteegh M, Kortbeek TM, Havelaar AH, van der Giessen JWB. Intervention strategies to reduce human Toxoplasma gondii disease burden. Clin Infect Dis. 2015;60:101-7.

3. Tenter AM, Heckeroth AR, Weiss LM. Toxoplasma gondii: from animals to humans. Int J Parasitol. 2000;30:1217-58.

4. Montoya JG, Liesenfeld O. Toxoplasmosis. Lancet. 2004;363:1965-76.

5. Bigna JJ, Tochie JN, Tounouga DN, Bekolo AO, Ymele NS, Youda EL, et al. Global, regional, and country seroprevalence of Toxoplasma gondii in pregnant women: a systematic review, modelling and meta-analysis. Sci Rep. 2020;10:12102

6. Dubey JP. Toxoplasmosis in sheep-the last 20 years. Vet Parasitol. 2009;163:1-14

7. Hiszczyńska-Sawicka E, Gatkowska JM, Grzybowski MM, Długońska $H$. Veterinary vaccines against toxoplasmosis. Parasitology. 2014;141:1365-78.

8. Stelzer S, Basso W, Benavides Silván J, Ortega-Mora LM, Maksimov P, Gethmann J, et al. Toxoplasma gondii infection and toxoplasmosis in farm animals: risk factors and economic impact. Food Waterborne Parasitol. 2019;15:e00037.

9. McAuley JB. Congenital Toxoplasmosis. J Pediatric Infect Dis Soc. 2014;3:30-5.

10. Dubey JP, Lindsay DS, Speer CA. Structures of Toxoplasma gondii tachyzoites, bradyzoites, and sporozoites and biology and development of tissue cysts. Clin Microbiol Rev. 1998;11:267-99. 
11. Dubey JP, Miller NL, Frenkel JK. The Toxoplasma gondii oocyst from cat feces. J Exp Med. 1970;132:636-62.

12. Halonen SK, Weiss LM. Toxoplasmosis. Handb Clin Neurol. 2013;114:125-45.

13. Weiss LM, Kim K. The development and biology of bradyzoites of Toxoplasma gondii. Front Biosci. 2000;5:391-405.

14. Ferguson DJ, Hutchison WM. The host-parasite relationship of Toxoplasma gondii in the brains of chronically infected mice. Virchows Arch A Pathol Anat Histopathol. 1987:411:39-43.

15. Schlüter D, Däubener W, Schares G, Groß U, Pleyer U, Lüder C. Animals are key to human toxoplasmosis. Int J Med Microbiol. 2014;304:917-29.

16. Chiebao DP, Bartley PM, Chianini F, Black LE, Burrells A, Pena HFJ, et al. Early immune responses and parasite tissue distribution in mice experimentally infected with oocysts of either archetypal or non-archetypal genotypes of Toxoplasma gondii. Parasitology. 2021;148:464-76.

17. Elsheikha HM. Congenital toxoplasmosis: priorities for further health promotion action. Public Health. 2008;122:335-53.

18. Gilbert R, Tan HK, Cliffe S, Guy E, Stanford M. Symptomatic Toxoplasma infection due to congenital and postnatally acquired infection. Arch Dis Child. 2006:91:495-8.

19. Jones JL, Lopez A, Wilson M, Schulkin J, Gibbs R. Congenital toxoplasmosis: a review. Obstet Gynecol Surv. 2001;56:296-305.

20. Capobiango JD, Breganó RM, Navarro IT, Rezende Neto CP, Casella AMB, Mori FMRL, et al. Congenital toxoplasmosis in a reference center of Paraná, southern Brazil. Braz J Infect Dis. 2014;18:364-71.

21. Flori P, Hafid J, Bourlet T, Raberin H, Genin C, Sung RTM. Experimental model of congenital toxoplasmosis in guinea-pigs: use of quantitative and qualitative PCR for the study of maternofetal transmission. J Med Microbiol. 2002;51:871-8.

22. Flori P, Hafid J, Thonier $\bigvee$, Bellete B, Raberin H, TranManhSung R. Parasite load in guinea pig foetus with real time PCR after maternofoetal transmission of Toxoplasma gondii. Parasite. 2003;10:133-40.

23. Vargas-Villavicencio JA, Besné-Mérida A, Correa D. Vertical transmission and fetal damage in animal models of congenital toxoplasmosis: a systematic review. Vet Parasitol. 2016;223:195-204.

24. Kressin M, Brehm R, Schnorr B. Embryologie der Haustiere. 7th ed. Stuttgart: Georg Thieme Verlag; 2019.

25. Workman AD, Charvet CJ, Clancy B, Darlington RB, Finlay BL. Modeling transformations of neurodevelopmental sequences across mammalian species. J Neurosci. 2013;33:7368-83.

26. Fietz SA, Kelava I, Vogt J, Wilsch-Bräuninger M, Stenzel D, Fish JL, et al. OSVZ progenitors of human and ferret neocortex are epithelial-like and expand by integrin signaling. Nat Neurosci. 2010;13:690-9.

27. Grigsby PL. Animal Models to study placental development and function throughout normal and dysfunctional human pregnancy. Semin Reprod Med. 2016;34:11-6.

28. Carter AM. Animal models of human pregnancy and placentation: alternatives to the mouse. Reproduction. 2020;160:129-43.

29. Mess A. The guinea pig placenta: model of placental growth dynamics. Placenta. 2007;28:812-5.

30. Haumont M, Delhaye L, Garcia L, Jurado M, Mazzu P, Daminet V, et al. Protective immunity against congenital toxoplasmosis with recombinant SAG1 protein in a guinea pig model. Infect Immun. 2000;68:4948-53.

31. Were SR, Bowman DD, Mohammed HO, Jenkins MB, Quimby FW, Horton $\mathrm{KM}$, et al. Transmission to guinea pigs of very low doses of oocysts of Toxoplasma gondii in drinking water. J Eukaryot Microbiol. 1999;46:71-2.

32. Henry L, Beverley J. Toxoplasmosis in rats and guinea-pigs. J Comp Pathol. 1977:87:97-102.

33. Grégoire A, Allard A, Huamán E, León S, Silva RM, Buff S, et al. Control of the estrous cycle in guinea-pig (Cavia porcellus). Theriogenology. 2012;78:842-7.

34. Burrells A, Taroda A, Opsteegh M, Schares G, Benavides J, Dam-Deisz C, et al. Detection and dissemination of Toxoplasma gondii in experimentally infected calves, a single test does not tell the whole story. Parasit Vectors. 2018;11:45

35. Dubey JP. Toxoplasmosis of animals and humans. 2nd ed. Boca Raton: CRC Press; 2016.

36. Schöniger S, Böttcher D, Theuß T, Schoon H-A. Expression of toll-like receptors 2, 4 and 6 in equine endometrial epithelial cells: a comparative in situ and in vitro study. Res Vet Sci. 2017;112:34-41.
37. Attig F, Spitzbarth I, Kalkuhl A, Deschl U, Puff C, Baumgärtner W, et al. Reactive oxygen species are key mediators of demyelination in canine distemper leukoencephalitis but not in Theiler's murine encephalomyelitis. Int J Mol Sci. 2019;20:3217

38. Gorka M, Schinköthe J, Ulrich R, Ciminski K, Schwemmle M, Beer M, et al. Characterization of experimental oro-nasal inoculation of Seba's short-tailed bats (Carollia perspicillata) with bat influenza A virus H18N11. Viruses. 2020;12:232.

39. Bier NS, Schares G, Johne A, Martin A, Nöckler K, Mayer-Scholl A. Performance of three molecular methods for detection of Toxoplasma gondii in pork. Food Waterborne Parasitol. 2019;14:38.

40. Belaz S, Gangneux J-P, Dupretz P, Guiguen C, Robert-Gangneux F. A 10-year retrospective comparison of two target sequences, REP-529 and B1, for Toxoplasma gondii detection by quantitative PCR. J Clin Microbiol. 2015;53:1294-300.

41. Talabani H, Asseraf M, Yera H, Delair E, Ancelle T, Thulliez P, et al. Contributions of immunoblotting, real-time PCR, and the Goldmann-Witmer coefficient to diagnosis of atypical toxoplasmic retinochoroiditis. J Clin Microbiol. 2009;47:2131-5.

42. Hoffmann B, Depner K, Schirrmeier H, Beer M. A universal heterologous internal control system for duplex real-time RT-PCR assays used in a detection system for pestiviruses. J Virol Methods. 2006;136:200-9.

43. Schares G, Dubey JP, Rosenthal B, Tuschy M, Bärwald A, Conraths FJ. Sensitive, quantitative detection of Besnoitia darlingi and related parasites in intermediate hosts and to assess felids as definitive hosts for known and as-yet undescribed related parasite species. Int J Parasitol Parasites Wildl. 2020;11:114-9.

44. Boyer K, Hill D, Mui E, Wroblewski K, Karrison T, Dubey JP, et al. Unrecognized ingestion of Toxoplasma gondii oocysts leads to congenital toxoplasmosis and causes epidemics in North America. Clin Infect Dis. 2011:53:1081-9.

45. Aguirre AA, Longcore T, Barbieri M, Dabritz H, Hill D, Klein PN, et al. The one health approach to toxoplasmosis: epidemiology, control, and prevention strategies. EcoHealth. 2019;16:378-90.

46. Müller J, Aguado-Martínez A, Ortega-Mora L-M, Moreno-Gonzalo J, Ferre I, Hulverson MA, et al. Development of a murine vertical transmission model for Toxoplasma gondii oocyst infection and studies on the efficacy of bumped kinase inhibitor (BKI)-1294 and the naphthoquinone buparvaquone against congenital toxoplasmosis. J Antimicrob Chemother. 2017;72:2334-41

47. Barbosa BF, Silva DAO, Costa IN, Pena JDO, Mineo JR, Ferro EAV. Susceptibility to vertical transmission of Toxoplasma gondii is temporally dependent on the preconceptional infection in Calomys callosus. Placenta. 2007;28:624-30.

48. Wright I. Transmission of Toxoplasma gondii across the guinea-pig placenta. Lab Anim. 1972;6:169-80.

49. Wong SY, Remington JS. Toxoplasmosis in pregnancy. Clin Infect Dis. 1994;18:853-61.

50. Dunn D, Wallon M, Peyron F, Petersen E, Peckham C, Gilbert R. Mother-tochild transmission of toxoplasmosis: risk estimates for clinical counselling Lancet. 1999:353:1829-33.

51. Wang T, Liu M, Gao X-J, Zhao Z-J, Chen X-G, Lun Z-R. Toxoplasma gondii: the effects of infection at different stages of pregnancy on the offspring of mice. Exp Parasitol. 2011;127:107-12.

52. Pusch L, Romeike B, Deckert M, Mawrin C. Persistent Toxoplasma bradyzoite cysts in the brain: incidental finding in an immunocompetent patient without evidence of a toxoplasmosis. Clin Neuropathol. $2009 \cdot 28 \cdot 210-2$

53. Evilsizor MN, Ray-Jones HF, Timothy W Ellis, JR, Lifshitz J, Ziebell JM. Microglia in experimental brain injury: implications on neuronal injury and circuit remodeling. In: Kobeissy FH, editor. Brain neurotrauma: molecular, neuropsychological, and rehabilitation aspects. Boca Raton (FL): CRC Press/Taylor \& Francis; 2015

\section{Publisher's Note}

Springer Nature remains neutral with regard to jurisdictional claims in published maps and institutional affiliations. 